\title{
Semi-Analytic Integration Method for Direct United Boundary-Domain Integro-Differential Equation Related to Dirichlet Problem
}

\author{
N. A. Mohamed, Member, IACSIT
}

\begin{abstract}
A semi-analytic integration method to handle the singularity of a parametrix concerning of direct united boundary-domain integro-differential Equation (BDIDE) related to the dirichlet boundary value problem for an elliptic Partial Differential Equation (PDE) with variable coefficient is presented in this paper. This approach can be an alternative to the Gauss-Laguerre quadrature formula to evaluate the integration with a kernel that deals with logarithmic singularity. The development of this method is inspired by the fact that the exact solution of an integral moves faster than its numerical solution. By using this approach, a result with high accuracy can still be obtained even with minimize numbers of the Gaussian quadrature points and thus reduce the numerical effort in the numerical integration.
\end{abstract}

Index Terms-Direct united boundary-domain integro-differential equation, Dirichlet problem, partial differential equation, semi-analytic integration method.

\section{INTRODUCTION}

It is widely known that a boundary-value problem (BVP) for a PDE can be reduced to a boundary-integral equation (BIE) provided that a fundamental solution for the PDE is known. The obtained BIE can then be solved numerically.

However, the fundamental solutions are known for many PDEs with constant coefficients and not generally available in an explicit form for PDEs with variable coefficients.

Unlike a fundamental solution, a parametrix (Levi function) is accessible in handling the variable coefficients cases. This approach will reduces the PDEs with variables coefficients not to a BIE but to a boundary-domain integral equation (BDIE) or a boundary-domain integro-differential equation (BDIDE), see e.g. [1]-[3].

We consider the following second-order linear elliptic PDE with variable coefficient $a(x)$ in a two-dimensional bounded domain $\Omega$,

$$
A u(x)=\sum_{i, j=1}^{2} \frac{\partial}{\partial x_{i}}\left[a(x) \frac{\partial u(x)}{\partial x_{j}}\right]=f(x), \quad x \in \Omega,
$$

with the Dirichlet boundary condition

Manuscript received February 18, 2014; revised April 24, 2014. This research was supported by the research grant RAGS, Vote No: 2013-0004-107-72.

N. A. Mohamed is with the Mathematical Department, Faculty of Science and Mathematics, Sultan Idris Education University (UPSI), 35900 Tanjong Malim, Perak Darul Ridzuan, Malaysia (e-mail: akmal.mohamed@fsmt.upsi.edu.my).

$$
u(x):=\bar{u}(x), \quad x \in \partial \Omega
$$

where $\partial \Omega$ is the boundary, $u(x)$ is the unknown function, while $f(x), \bar{u}(x)$ and $a(x)>0$ are prescribed functions.

A parametrix

$$
P(x, y)=\frac{\ln |x-y|}{2 \pi a(y)}, \quad x, y \in \mathbb{R}^{2},
$$

for PDE (1) with variable coefficient, yielded from the fundamental solution for the same equation but with 'frozen' coefficient $a(x)=a(y)$. Here the radius $r$ is given below.

$$
r=|x-y|=\sqrt{\left(x_{i}-y_{i}\right)\left(x_{i}-y_{i}\right)} .
$$

The parametrix (2) satisfies equation

$$
A_{x} P(x, y)=\delta(x-y)+R(x, y),
$$

where $\delta(x-y)$ is the Dirac delta function, and the remainder $R$ is as follows.

$$
R(x, y)=\frac{1}{2 \pi a(y)} \sum_{i=1}^{2} \frac{x_{i}-y_{i}}{|y-x|} \frac{\partial a(x)}{\partial x_{i}}, \quad x, y \in \mathbb{R}^{2} .
$$

Note that the remainder $R$ [3] has only a weak singularity at $x-y$.

Let also denote that

$$
\begin{gathered}
T u(x)=\sum_{j=1}^{2} a(x) v_{j}(x) \frac{\partial u(x)}{\partial x_{j}} \\
T_{x} P(x, y)=\sum_{j=1}^{2} a(x) v_{j}(x) \frac{\partial P(x, y)}{\partial x_{j}}=\sum_{j=1}^{2} \frac{a(x) v_{j}(x)\left(x_{j}-y_{j}\right)}{2 \pi a(y) r^{2}} .
\end{gathered}
$$

Here $v(x)=\left(v_{1}(x), v_{2}(x)\right)$ is the outward normal to $\Omega$.

As described in [1], [4] for united formulation, the direct united boundary-domain integro-differential equation (BDIDE) for the Dirichlet problem with respect to the unknown function $u$ is given below.

$$
\begin{aligned}
& c(y) u(y)+\int_{\Omega} R(x, y) u(x) \mathrm{d} \Omega(x) \\
& +\int_{\partial \Omega} P(x, y) T u(x) \mathrm{d} \Gamma(x)=\int_{\partial \Omega} \bar{u}(x) T_{x} P(x, y) \mathrm{d} \Gamma(x)(3) \\
& +\int_{\Omega} P(x, y) f(x) \mathrm{d} \Omega(x), \quad y \in \bar{\Omega}
\end{aligned}
$$

where 


$$
c(y)=\left\{\begin{array}{cc}
1 & \text { if } y \in \Omega^{+}, \\
0 & \text { if } y \in \Omega^{-} \\
\alpha(y) / 2 \pi & \text { if } y \in \partial \Omega
\end{array}\right.
$$

and $\alpha(y)$ is an interior angle at a corner point $y$ of the boundary $\partial \Omega$. If $\partial \Omega$ is a smooth boundary, then $\alpha(y)=\pi$ such that $c(y)=1 / 2$. The first integral in the right hand side of (3) is understood in the Cauchy principal value sense if $y \in \partial \Omega$, see e.g. [5].

Some analysis of the direct boundary-domain integral equations with variable coefficient can be found in e.g. [6] and [7].

In [8], [9] the system of equations obtained from discretized BDIE related to Neumann problem for PDE with variable coefficient was solved by the direct (LU decomposition) method and Neumann iteration method. In [9], the fast convergence of the iterative method is investigated by calculated the eigen-values of the obtained algebraic systems approximating the eigen-values of the BDIE. In both papers [8], [9], the boundary integral that consists of Parametrix is evaluated by using Gauss-Laguerre quadrature formula.

In this paper, we focus on the BDIDE related to Dirichlet problem for PDE with variable coefficient as given in (4). The BDIDE in (4) is consisting of several integrals that involve singularity. The first integral in the right hand side of (3) is understood in the Cauchy principal value sense if $y \in \partial \Omega$. The singularity of the domain integrals i.e. the first integral in the left hand side of (4) and the second integral in the right hand side of (4) are both can be treated by using Duffy transformation, see. e.g. [5]. The boundary integral that consists of Parametrix i.e. the second integral in the left can be handled by using Gauss-Laguerre quadrature formula. However, the approach might require considerable numbers of the Gaussian quadrature points in order to achieve a high accuracy result. In this paper, we propose a semi-analytic integration method to avoid the use of Gauss-Laguerre quadrature formula in calculating the second integral in the left hand side of (3).

\section{DisCRETIZATION OF THE BDIDE AND THE SEMI-ANALYTIC INTEGRATION METHOD}

\section{A. Discretization of the BDIDE}

We discretised the domain $\Omega$ by a mesh of $M$ iso-parametric quadrilateral bilinear domain elements i.e. $\bar{\Omega}=\bigcup_{m}^{M} e_{m}, e_{k} \cap e_{m}=\varnothing, k \neq m$, we can write the Cartesian coordinates of a point on domain element $e_{m} \subset \Omega$ in terms of the intrinsic coordinates $\left(\xi_{1}, \xi_{2}\right)=: \xi$ on the reference square $-1 \leq \xi_{1} \leq 1,-1 \leq \xi_{2} \leq 1$ as

$$
x(\xi)=\sum_{N=1}^{4} \Phi_{N}(\xi) X^{m N},
$$

where $\Phi_{N}(\xi)$ are the local shape functions,

$$
\begin{aligned}
& \Phi_{1}(\xi)=\left(1-\xi_{1}\right)\left(1-\xi_{2}\right) / 4, \\
& \Phi_{2}(\xi)=\left(1+\xi_{1}\right)\left(1-\xi_{2}\right) / 4, \\
& \Phi_{3}(\xi)=\left(1+\xi_{1}\right)\left(1+\xi_{2}\right) / 4, \\
& \Phi_{4}(\xi)=\left(1-\xi_{1}\right)\left(1+\xi_{2}\right) / 4,
\end{aligned}
$$

and $X^{m N}, N=1, \ldots, 4$ are the vertices for each domain element $e_{m}$.

The unknown function $u(x)$ at any point $x \in \bar{\Omega}$ is interpolated over its values $u\left(x^{j}\right)$ at the global nodes $x^{j}$ such that

$$
u(x)=\sum_{j} \phi_{j}(x) u\left(x^{j}\right), \quad x, x^{j} \in \Omega \cup \partial \Omega,
$$

where $\phi_{j}(x)$ are the global shape functions satisfying the so-called $\delta$-property i.e. $\phi_{j}\left(x_{k}\right)=\delta_{j k}$,

$$
\phi_{j}(x)=\left\{\begin{array}{cl}
\Phi_{N}\left(\xi^{m}(x)\right) & \text { if } \quad x \in \bar{e}_{m}, x^{j} \in\left\{X^{m N}\right\}_{N=1}^{4}, \\
0 & \text { if } x \in \bar{e}_{m}, x^{j} \notin\left\{X^{m N}\right\}_{N=1}^{4},
\end{array}\right.
$$

where $\xi^{m}(x)$ are the functions inverse to (4). See e.g. [9].

The boundary $\partial \Omega$ is dicretized with $L$ continuous linear iso-parametric elements, $\partial \Omega=\bigcup_{l}^{L} \partial \Omega_{l}, \quad$ where $\partial \Omega_{1}, \partial \Omega_{2}, \ldots, \partial \Omega_{L}$ are the outer sides of the corresponding domain elements $e_{m}$.

The Cartesian coordinates of a point on a boundary element $\partial \Omega_{l} \subset \partial \Omega$ with the intrinsic coordinate $\eta$ on the reference segment $-1 \leq \eta \leq 1$ are as follows.

$$
x(\eta)=\sum_{n=1}^{2} \Psi_{n}(\eta) X^{l n},
$$

where $\Psi_{n}(\eta)$ are the local one-dimensional shape functions, that are the traces of the two-dimensional shape functions $\Phi_{N}(\xi)$ :

$$
\Psi_{1}(\eta)=\frac{1}{2}(1-\eta), \quad \Psi_{2}(\eta)=\frac{1}{2}(1+\eta), \quad-1 \leq \eta \leq 1,
$$

and $X^{l n}, n=1,2$, are the endpoints for each boundary element $\partial \Omega_{l}$.

Equation (3) is equivalent to the following equation:

$$
\begin{aligned}
u(y) & +\int_{\Omega} R(x, y) u(x) \mathrm{d} \Omega(x) \\
& +\int_{\partial \Omega} P(x, y) T u(x) \mathrm{d} \Gamma(x)=(1-c(y)) \bar{u}(y) \\
& +\int_{\partial \Omega} \bar{u}(x) T_{x} P(x, y) \mathrm{d} \Gamma(x) \\
& +\int_{\Omega} P(x, y) f(x) \mathrm{d} \Omega(x), \quad y \in \bar{\Omega} .
\end{aligned}
$$

We then apply interpolation to (6). Setting $J$ number of 
nodes and placing the collocation points $x^{i}$ for $x^{j} \in \bar{\Omega}$ at all nodes $x^{j} \in \bar{\Omega}$, yields the following system of $J$ linear algebraic equations for $J$ unknowns $u\left(x^{i}\right)$.

$$
\begin{aligned}
u\left(x^{i}\right) & +\sum_{x^{i} \in \Omega} K_{i j}^{D} u\left(x^{j}\right)=\left(1-c\left(x^{i}\right)\right) \bar{u}\left(x^{i}\right) \\
& +Q_{i}^{D}+D_{i}^{D}, \quad x^{i} \in \bar{\Omega}, x^{j} \in \bar{\Omega}, j=1,2, \ldots, J,
\end{aligned}
$$

where $K_{i j}^{D}, Q_{i}^{D}$ and $D_{i}^{D}$ are defined as in (8)-(10).

$$
\begin{aligned}
& K_{i j}^{D}=\int_{\Omega} \phi_{j}(x) R(x, y) \mathrm{d} \Omega(x) \\
& +\int_{\partial \Omega} P(x, y) a(x)\left(\frac{\partial \phi_{j}(x)}{\partial v(x)}\right) \mathrm{d} \Gamma(x) \\
& =\sum_{m=1}^{M} \int_{\Omega_{m}} \phi_{j}(x) R\left(x, x^{i}\right) \mathrm{d} \Omega(x) \\
& +\sum_{l=1}^{L} \int_{\partial \Omega_{l}} P\left(x, x^{i}\right) a(x)\left(\frac{\partial \phi_{j}(x)}{\partial v(x)}\right) \mathrm{d} \Gamma(x) \\
& =\sum_{x^{j} \in \Omega_{m}} \int_{\Omega_{m}} \phi_{j}(x) R\left(x, x^{i}\right) \mathrm{d} \Omega(x) \\
& +\sum_{\left\{x^{j} \in \Omega_{m}: \Omega_{m}\right\} \supset \partial \Omega_{i}} \int_{\partial \Omega_{t}} P\left(x, x^{i}\right) a(x)\left(\frac{\partial \phi_{j}(x)}{\partial v(x)}\right) \mathrm{d} \Gamma(x), \\
& Q_{i}^{D}=\int_{\partial \Omega} \bar{u}(x) T_{x} P(x, y) \mathrm{d} \Gamma(x) \\
& =\sum_{l=1}^{L} \int_{\partial \Omega_{l}} \bar{u}(x) T_{x} P\left(x, x^{i}\right) \mathrm{d} \Gamma(x), \\
& D_{i}^{D}=\int_{\Omega} P(x, y) f(x) \mathrm{d} \Omega(x) \\
& =\sum_{m=1}^{M} \int_{\Omega_{m}} P\left(x, x^{i}\right) f(x) \mathrm{d} \Omega(x) .
\end{aligned}
$$

Changing the integration variables to the intrinsic coordinates, we can then write (8)-(10) as

$$
\begin{aligned}
K_{i j}^{D} & =\sum_{x^{j} \in \Omega_{m}} G_{N(j, m), i}^{m}+\sum_{\left\{x^{j} \in \Omega_{m}: \Omega_{m}\right\} \supset \partial \Omega_{i}} A_{N(j, m), i}^{l} \\
Q_{i}^{D} & =\sum_{l=1}^{L} F_{i}^{l} \\
D_{i} & =\sum_{m=1}^{M} F_{i}^{m}
\end{aligned}
$$

where $n(j, l)$ is the local number of the node $x^{j}$ on the boundary element $\partial \Omega_{l}, N(j, m)$ is the local number of the node $x^{j}$ on the domain element $e_{m}$, and $G_{N, i}^{m}, A_{N, i}^{l}, F_{i}^{l}$ and $H_{i}^{m}$ are given as follows:

$$
G_{N, i}^{m}=\int_{-1}^{1} \int_{-1}^{1} \Phi_{N}(\xi) R\left(x(\xi), x^{i}\right) J_{m 2}(\xi) \mathrm{d} \xi_{1} \mathrm{~d} \xi_{2},
$$

$$
\begin{gathered}
A_{N, i}^{l}=\int_{-1}^{1} P\left(x(\eta), x^{i}\right) a(x(\eta)) \\
\left(\left.\sum_{p=1}^{2} \sum_{k=1}^{2} \frac{\partial \Phi_{N}}{\partial \xi_{k}} \frac{\partial \xi_{k}}{\partial x_{p}}\right|_{\xi=\xi(\eta)} v_{p}(x(\eta))\right) J_{l 1}(\eta) \mathrm{d} \eta, \\
F_{i}^{l}=\int_{-1}^{1} \bar{u}(x(\eta)) T_{x} P\left(x(\eta), x^{i}\right) J_{l 1}(\eta) \mathrm{d} \eta, \\
H_{i}^{m}=\int_{-1}^{1} \int_{-1}^{1} P\left(x(\xi), x^{i}\right) f(x(\xi)) J_{m 2}(\xi) \mathrm{d} \xi_{1} \mathrm{~d} \xi_{2} .
\end{gathered}
$$

Here $J_{m 2}$ and $J_{l 1}$ represent the Jacobians of the transforms (4) and (5), respectively.

When the collocation point $x^{i}$ is not a vertex of the integration element, the integrals in (11)-(14) are evaluated by the Gauss-Legendre quadrature formulas,

$$
\begin{aligned}
& \int_{-1}^{1} f(\eta) \mathrm{d} \eta=\sum_{p=1}^{\hat{i}} W_{p} f\left(\eta_{p}\right), \\
& \int_{-1}^{1} \int_{-1}^{1} f(\xi) \mathrm{d} \xi_{1} \mathrm{~d} \xi_{2}=\sum_{q=1}^{\hat{j}} \sum_{p=1}^{\hat{i}} W_{p} W_{q} f\left(\xi_{1 p}, \xi_{2 q}\right),
\end{aligned}
$$

where $\hat{i}$ and $\hat{j}$ are the numbers of quadrature points, $\eta_{p}, \xi_{1 p}$ and $\xi_{2 p}$ are the quadrature point coordinates, while $W_{p}$ and $W_{q}$ are the quadrature weights associated to point $p$ and $q$, respectively (see e.g. [5]).

\section{B. Semi-Analytic Integration Method}

When a collocation point $x^{i}$ is a vertex of the integration element, the kernels of the integrals (11), (12) and (14) are weakly singular at collocation points, thus need a special treatment.

The integrals (12) with the kernel involving $\ln (r)$ can be evaluated numerically by using the Gauss-Laguerre quadrature formula to handle the influence of the singularity $\ln (r)$ when the collocation point $x^{i}$ is near to the integration element $x^{j}$. Instead, we can also use the proposed semi -analytic integration formula as explained below. The semi-analytic integration formula is arranged as follows.

$$
\begin{aligned}
& \int_{\partial \Omega_{t}} P\left(x, x^{i}\right) a(x)\left(\frac{\partial \phi_{j}(x)}{\partial v(x)}\right) \mathrm{d} \Gamma(x) \\
& =\int_{\partial \Omega_{t}}\left[P\left(x, x^{i}\right) a(x)\left(\frac{\partial \phi_{j}(x)}{\partial v(x)}\right)-g_{i j l}\right] \mathrm{d} \Gamma(x)+G_{i j l},
\end{aligned}
$$

where

$$
\begin{aligned}
g_{i j l} & =\left(\frac{s_{2}-s}{s_{2}-s_{1}}\right) P\left(x, x^{i}\right) a\left(x\left(s_{1}\right)\right) \frac{\partial \phi_{j}(x)}{\partial v(x)} \\
& +\left(\frac{s-s_{1}}{s_{2}-s_{1}}\right) P\left(x, x^{i}\right) a\left(x\left(s_{2}\right)\right) \frac{\partial \phi_{j}(x)}{\partial v(x)},
\end{aligned}
$$




$$
\begin{aligned}
& G_{i j l}=\int_{\partial \Omega_{l}} g_{i j l} \mathrm{~d} s \\
& =\int_{\partial \Omega_{l}}\left(\frac{s_{2}-s}{s_{2}-s_{1}}\right) P\left(x, x^{i}\right) a\left(x\left(s_{1}\right)\right) \frac{\partial \phi_{j}\left(x\left(s_{1}\right)\right)}{\partial v(x)} \mathrm{d} s \\
& +\int_{\partial \Omega_{l}}\left(\frac{s-s_{1}}{s_{2}-s_{1}}\right) P\left(x, x^{i}\right) a\left(x\left(s_{2}\right)\right) \frac{\partial \phi_{j}\left(x\left(s_{2}\right)\right)}{\partial v(x)} \mathrm{d} s .
\end{aligned}
$$

Since

$$
\begin{aligned}
& \left(s_{2}-s\right)=\frac{\left(s_{2}-s_{1}\right)}{2}(1-\eta), \\
& \left(s-s_{1}\right)=\frac{\left(s_{2}-s_{1}\right)}{2}(1-\eta),
\end{aligned}
$$

one can write (15) and (16) as

$$
\begin{aligned}
g_{i j l} & =\frac{(1-\eta)}{2} P\left(x, x^{i}\right) a\left(x\left(s_{1}\right)\right) \frac{\partial \phi_{j}(x)}{\partial v(x)} \\
& +\frac{(1+\eta)}{2} P\left(x, x^{i}\right) a\left(x\left(s_{2}\right)\right) \frac{\partial \phi_{j}(x)}{\partial v(x)}, \\
G_{i j l} & =\int_{\partial \Omega_{i}} g_{i j l} \mathrm{~d} s \\
= & \int_{\partial \Omega_{t}} \frac{(1-\eta)}{2} P\left(x, x^{i}\right) a\left(x\left(s_{1}\right)\right) \frac{\partial \phi_{j}\left(x\left(s_{1}\right)\right)}{\partial v(x)} \mathrm{d} s \\
+ & \int_{\partial \Omega_{i}} \frac{(1+\eta)}{2} P\left(x, x^{i}\right) a\left(x\left(s_{2}\right)\right) \frac{\partial \phi_{j}\left(x\left(s_{2}\right)\right)}{\partial v(x)} \mathrm{d} s \\
& =a\left(x\left(s_{1}\right)\right) \frac{\partial \phi_{j}\left(x\left(s_{1}\right)\right)}{\partial v(x)} \\
& \int_{-1}^{1} \frac{(1-\eta)}{2} P\left(x, x^{i}\right) a\left(x\left(s_{1}\right)\right) \frac{d s}{d \eta} \mathrm{d} \eta \\
& +a\left(x\left(s_{2}\right)\right) \frac{\partial \phi_{j}\left(x\left(s_{2}\right)\right)}{\partial v(x)} \\
& \int_{-1}^{1} \frac{(1+\eta)}{2} P\left(x, x^{i}\right) a\left(x\left(s_{2}\right)\right) \frac{d s}{d \eta} \mathrm{d} \eta .
\end{aligned}
$$

Defining

$$
\begin{gathered}
g_{A 1}=\int_{-1}^{1} \frac{(1-\eta)}{2} P\left(x, x^{i}\right) a\left(x\left(s_{1}\right)\right) \frac{d s}{d \eta} \mathrm{d} \eta, \\
g_{A 2}=\int_{-1}^{1} \frac{(1+\eta)}{2} P\left(x, x^{i}\right) a\left(x\left(s_{2}\right)\right) \frac{d s}{d \eta} \mathrm{d} \eta,
\end{gathered}
$$

we can then write (18) as

$$
\begin{aligned}
G_{i j l} & =a\left(x\left(s_{1}\right)\right) \frac{\partial \phi_{j}\left(x\left(s_{1}\right)\right)}{\partial v(x)} g_{A 1} \\
& +a\left(x\left(s_{2}\right)\right) \frac{\partial \phi_{j}\left(x\left(s_{2}\right)\right)}{\partial v(x)} g_{A 2} .
\end{aligned}
$$

The integrals $g_{A 1}$ and $g_{A 2}$ in (19) and (20) are calculated analytically. The radius $r$ can be written as $r=\sqrt{h^{2}+(d-s)^{2}}$, where $h, d$ and $s$ are defined as in (21)-(23) below.

$$
\begin{gathered}
h=\frac{\left|\vec{W}_{1} \times \vec{W}_{2}\right|}{W_{2}}=\frac{W_{1} W_{2} \sqrt{1-\left(\hat{W}_{1} \cdot \hat{W}_{2}\right)^{2}}}{W_{2}} \\
=W_{1}(1-\tilde{e}), \\
d=W_{2} \cos \theta=\vec{W}_{2} \cdot \hat{W}_{1}=\frac{\vec{W}_{1} \cdot \vec{W}_{2}}{W_{1}}=\frac{W_{3}}{W_{2}}, \\
\quad s=\frac{W_{2}}{2}(\eta+1),
\end{gathered}
$$

where

$$
\begin{gathered}
W_{1}=\left|x^{i}-x\left(s_{l 1}\right)\right|, \\
W_{2}=\left|x\left(s_{l 2}\right)-x\left(s_{l 1}\right)\right|, \\
W_{3}=\left(x^{i}-x\left(s_{l 1}\right)\right) \cdot\left(x\left(s_{l 2}\right)-x\left(s_{l 1}\right)\right), \\
\tilde{e}=\left(\hat{W}_{1} \cdot \hat{W}_{2}\right)^{2}=\left(\frac{\vec{W}_{1} \cdot \vec{W}_{2}}{W_{1} W_{2}}\right)^{2}=\left(\frac{W_{3}}{W_{1} W_{2}}\right)^{2},
\end{gathered}
$$

Here $\widehat{W}_{1}$ and $\hat{W}_{2}$ are the unit vectors.

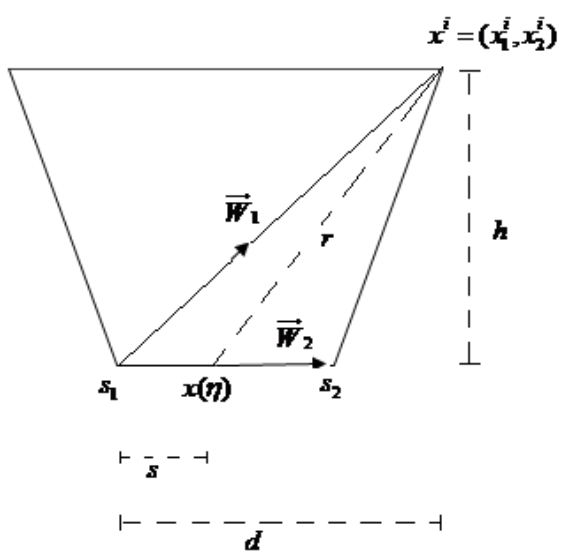

Fig. 1. Illustrations of the notations used in describing this semi-analytic integration method.

Therefore we can write $g_{A 1}$ and $g_{A 2}$ in (19) and (20) as

$$
\begin{aligned}
& g_{A 1}=\int_{-1}^{1}\left(\frac{1-\eta}{2}\right)\left(\frac{1}{2}\right)\left(\frac{\ln \left[h^{2}+(d-s)^{2}\right]}{2 \pi a\left(x^{i}\right)}\right) \frac{d s}{d \eta} \mathrm{d} \eta, \\
& g_{A 2}=\int_{-1}^{1}\left(\frac{1+\eta}{2}\right)\left(\frac{1}{2}\right)\left(\frac{\ln \left[h^{2}+(d-s)^{2}\right]}{2 \pi a\left(x^{i}\right)}\right) \frac{d s}{d \eta} \mathrm{d} \eta .
\end{aligned}
$$

The analytic solutions for integrals $g_{A 1}$ and $g_{A 2}$ are calculated exactly by using Mathematica 5.1 as given in equations (24) and (25) below. 


$$
\begin{gathered}
g_{A 1}=\frac{J_{l 1}(\eta)\left(h_{1}+h_{2}+h_{3}+h_{4}\right)}{4 \pi a\left(x^{i}\right) W_{2}^{4}}, \\
g_{A 2}=\frac{J_{l 1}(\eta)\left(f_{1}+f_{2}+f_{3}+f_{4}\right)}{4 \pi a\left(x^{i}\right) W_{2}^{4}},
\end{gathered}
$$

where

$$
\begin{aligned}
J_{l 1}(\eta)= & \frac{d s}{d \eta}, \\
h_{1}= & -3 W_{2}^{4}+2 W_{2}^{2} W_{3}+4\left(W_{2}^{2}-W_{3}\right) \sqrt{W_{1}^{2} W_{2}^{2}-W_{3}^{2}} \\
& \operatorname{ArcTan}\left[\frac{\left(W_{2}^{2}-W_{3}\right)}{\sqrt{W_{1}^{2} W_{2}^{2}-W_{3}^{2}}}\right], \\
h_{2}= & \left(W_{2}^{2}-W_{3}\right) \sqrt{W_{1}^{2} W_{2}^{2}-W_{3}^{2}} \\
& \operatorname{ArcTan}\left[\frac{W_{3}}{\left.\sqrt{W_{1}^{2} W_{2}^{2}-W_{3}^{2}}\right],}\right. \\
h_{3}= & \left(W_{1}^{2} W_{2}^{2}+2 W_{2}^{2} W_{3}-2 W_{3}^{2}\right) \ln \left[W_{1}^{2}\right], \\
h_{4}= & \left(-W_{1}^{2} W_{2}^{2}+W_{2}^{4}-2 W_{2}^{2} W_{3}+2 W_{3}^{2}\right) \\
& \ln \left[W_{1}^{2}+W_{2}^{2}-2 W_{3}\right], \\
f_{4}= & \left(W_{1}^{2} W_{2}^{2}+W_{2}^{4}-2 W_{3}^{2}\right) \ln \left[W_{1}^{2}+W_{2}^{2}-2 W_{3}\right] . \\
f_{1}= & -W_{2}^{4}-2 W_{2}^{2} W_{3}+4 W_{3} \sqrt{W_{1}^{2} W_{2}^{2}-W_{3}^{2}} \\
& \operatorname{ArcTan}\left[\frac{\left(W_{2}^{2}-W_{3}\right)}{\sqrt{W_{1}^{2} W_{2}^{2}-W_{3}^{2}}}\right],
\end{aligned}
$$

The analytic solutions for integrals $g_{A 1}$ and $g_{A 2}$ in (24) and (25) are uncertainty of the type $0 / 0$ when $x^{i}=s_{1}$ and $x^{i}=s_{2}$. Therefore, when $x^{i}=s_{1}$, by taking the limit as $W_{1} \rightarrow 0$, we obtain

$$
\begin{aligned}
& g_{A 1}=\left(\frac{1}{4 \pi a\left(x^{i}\right)}\right) J_{l 1}(\eta)\left(-3+\ln \left[W_{2}^{2}\right]\right), \\
& g_{A 2}=\left(\frac{1}{4 \pi a\left(x^{i}\right)}\right) J_{l 1}(\eta)\left(-1+\ln \left[W_{2}^{2}\right]\right) .
\end{aligned}
$$

When $x^{i}=s_{2}$, by taking the limit as $W_{1} \rightarrow W_{2}$, we have

$$
\begin{aligned}
& g_{A 1}=\left(\frac{1}{4 \pi a\left(x^{i}\right)}\right) J_{l 1}(\eta)\left(-1+\ln \left(W_{2}^{2}\right)\right), \\
& g_{A 2}=\left(\frac{1}{4 \pi a\left(x^{i}\right)}\right) J_{l 1}(\eta)\left(-3+\ln \left(W_{2}^{2}\right)\right) .
\end{aligned}
$$

In handling the singularity for the domain integrals (11) and (14), we can split the square reference element into triangular sub-elements and apply the Duffy transformation, see, e.g. [5].

System (7) can now be solved by any numerical method for solving linear algebraic systems.

\section{CONCLUSION}

As for the conclusion, we have introduced one approach in handling the integration that involves singularity for the parametrix $P(x, y)$. The proposed method lies on the idea that the exact solution of an integral moves faster than the its numerical solution. Therefore, this semi-analytic integration method will make the numerical solution approach the exact solution of the respected integral's solution closer than the standard numerical approach. Calculating (12) exactly is also possible but of cause not a good idea since the parametrix $P(x, y)$ that appears in (12) is consisting of variable $a(x)$ which might be different for different cases of BVPs. This semi-analytic integration is then a good way to avoid calculating (12) each time whenever we have different values of $a(x)$.

Some numerical tests also indicate that this semi-analytic integration method does produce higher accuracy than those when we calculate the integral involving a Parametrix by using Gauss- Laguerre formula to handle the singularity of a logarithmic function. However, no numerical result will be presented in this paper.

\section{REFERENCES}

[1] S. E. Mikhailov, "Localized boundary-domain integral formulations for problems with variable coefficients," Engng Anal. Boundary Elem, vol. 26, pp. 681-690, 2002.

[2] S. E. Mikhailov and I. S. Nakhova, "Mesh-based numerical implementation of the localized boundary-domain integral-equation method to a variable-coefficient Neumann problem," J. Eng. Math., vol. 51, pp. 251-259, 2005.

[3] S. E. Mikhailov, "Localized direct boundary domain integro-differential formulations for scalar nonlinear boundary-value problems with variable coefficients," J. Eng. Math., vol. 51, no. 3, pp. 283-302, 2005.

[4] S. E. Mikhailov, "Analysis of united boundary-domain integro-differential and integral equations for a mixed BVP with variable coefficient," Math. Methods in Applied Sciences, vol. 29, pp. 715-739, 2002.

[5] G. Beer, Programming the Boundary Element Method, West Sussex: John Wiley \& Wiley, 2001, ch. 6, pp. 119-130.

[6] O. Chkadua, S. E. Mikhailov, and D. Natroshvili, "Analysis of direct boundary-domain integral equations for a mixed BVP with variable coefficient, I: Equivalence and invertibility," Journal of Integral Equations and Applications, vol. 21, pp. 499-543, 2009.

[7] O. Chkadua, S. E. Mikhailov, and D. Natroshvili, "Analysis of segregated boundary-domain integral equations for variable-coefficient problems with cracks," Numer. Meth. for PDEs, vol. 27, no. 1, pp. 121-140, 2011.

[8] S. E. Mikhailov and N. A. Mohamed, "Iterative solution of boundary-domain integral equation for $\{\mathrm{BVP}\}$ with variable 
coefficient," in Proc. the 8th UK Conference on Boundary Integral Methods D. Lesnic ed., pp. 127-134, UK: Leeds University Press, ISBN 978085316 2957, 2011

[9] S. E. Mikhailov and N. A. Mohamed, "Numerical solution and spectrum of boundary-domain integral equation for the Neumann BVP with a variable coefficient," International Journal of Computer Mathematics, pp. 1-17, DOI: 10.1080/00207160.2012.679733, 2012

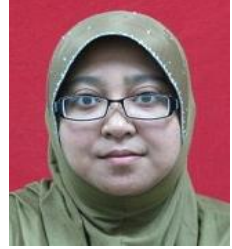

Nurul Akmal Mohamed was born in Kelantan, Malaysia, in 1983. She received her B.sc and M.sc from University of Technology Malaysia, Skudai, Johore, Malaysia in 2005 and 2007, respectively. Later she obtained her P.hD in mathematics from Brunel University, West London, United Kingdom in the year 2013. She is currently a senior lecturer at Sultan Idris Education University, Perak, Malaysia. Her research interests include analysis and numerical solution of partial differential equations and complex analysis. 